\title{
Guest Editorial Special Issue on Time-delay Systems and Their Applications
}

In many real systems such as electrical/electronics systems, chemical/process control systems, communication networks, environment/population dynamics, biological systems, and financial systems, time delays often appear in the states, the control inputs, or the measurements. Time-delay systems are of infinite dimensions in nature and their property leads huge complexity in the analysis of the systems. Also, it is well known that the existence of time delays often causes undesirable dynamic behaviors, deteriorates the performance, and even destroys the stability. In this regard, during the last three decades, the analysis and synthesis of time-delay systems and their applications in the real world have been received a great deal of attention in societies of control engineering and mathematics and thus numerous papers have been published in the literature.

The overall aims of this special issue are two-fold: (1) to provide an up-to-date overview of recent research trends on time-delay systems and (2) to offer the advanced and practical methodologies to deal with time delays in real practical applications. The eight papers in this special issue are invited by prominent specialists who are well known in the field of time-delay systems.

The first paper entitled "Auxiliary Function-based Integral/Summation Inequalities: Application to Continuous/Discrete Time-delay Systems" has developed novel integral/summation inequalities via some intermediate terms called auxiliary functions. It has been shown that the proposed inequalities turn into the various existing inequalities by appropriately selecting the auxiliary functions.

In the second paper entitled "Enhancement on Stability Criteria for Linear Systems with Interval Time-varying Delays," By constructing a suitable augmented Lyapunov-Krasovskii functional and utilizing Wirtinger-based integral inequality, two sufficient conditions for guaranteeing the asymptotic stability of the concerned systems are derived within the framework of linear matrix inequalities.

In the work entitled "Stability Analysis for T-S Fuzzy Systems with Time-varying Delay via Free-matrix-based Integral Inequality," the authors have studied the stability problem for T-S fuzzy systems with time-varying delay by using the LKF approach together with the free-matrix-based integral inequality. It is noted that the fuzzy-weighting matrix variables aiming at different fuzzy subsystems are employed to reduce the conservatism.

In the fourth paper entitled "Local Stability and Local Stabilization of Discrete-time T-S Fuzzy Systems with TimeDelay," in order to estimate the domain of attraction of the time-delay systems, the so-called fuzzy Lyapunov-Krasovskii functionals are used to characterize the subsets of the domain of attraction as sublevel sets of the Lyapunov-Krasovskii functionals by utilization of the information of variation rates of fuzzy membership functions.

The fifth paper entitled "Dissipative based Adaptive Reliable Sampled-data Control of Time-Varying Delay Systems" is concerned with the problem of dissipative based adaptive reliable controller for a class of time delay systems subject to actuator failures and time-varying sampling with a known upper bound on the sampling intervals.

In the sixth paper entitled "Constrained Model Predictive Control for Time-varying Delay Systems: Application to an Active Car Suspension," a feasible state feedback is developed by minimizing an upper bound of the 'worst-case' quadratic objective function over an infinite horizon subject to constraints on inputs. The design method is applied to a quarter-vehicle model.

The work entitled "Dynamic Output-feedback $\mathscr{H}_{\infty}$ Control for Active Half-vehicle Suspension Systems with Timevarying Input Delay" presents a scheme of multi-objective synthesis is used to design a dynamic controller such that the closed-loop suspension system is asymptotically stable with guaranteed performance in the $\mathscr{H}_{\infty}$ sense. 
In the last paper entitled "Mean Square Consensus of Multi-agent Systems with Multiplicative Noises and Time Delays under Directed Fixed Topologies," by considering general network under directed fixed topologies, a consensus protocol that take into account both the multiplicative noises and time delays is proposed and sufficient conditions composed of the upper bounds of consensus gain and time-delays are obtained by use of the tools from stochastic differential delay equation, martingale theory and stochastic inequality.

\section{Acknowledgments}

The guest editors would like to thank all the authors for their great contributions of this special issue. We are also grateful to Editor-in-Chief, Prof. Yong Hoon Joo and the Managing Editor, Ms. Jinyoung You, of the International Journal of Control, Automation, and Systems for providing them with the opportunity to organize the issue.

\section{Guest Editors}

Ju H. Park

Department of Electrical Engineering

Yeungnam Univeristy

Kongsan, Republic of Korea

Email: jessie@ynu.ac.kr

PooGyeon Park

Department of Electronic and Electrical Engineering

Pohang University of Science and Technology

Pohang, Republic of Korea

Email:ppg@postech.ac.kr 